\title{
Prediction of Cryptocurrency Price Index Using Artificial Neural Networks: A Survey of the Literature
}

\author{
Sina E. Charandabi and Kamyar Kamyar
}

\section{ABSTRACT}

\begin{abstract}
This paper initially presents a brief overview of the cryptocurrency and its history. We discuss the novel nature of literature attempting to create hybrid artificial neural network models to predict prices of cryptocurrency. For the remaining majority of the paper, we present the details of various hybrid artificial neural networks that have successfully been implemented to predict cryptocurrency prices in the form of a survey. Comparison of methods and results follow in the results section.
\end{abstract}

Keywords: artificial neural networks, cryptocurrency prices, prediction survey.

\author{
Submitted : October 10, 2021 \\ Published : November 09, 2021 \\ ISSN: 2507-1076 \\ DOI: $10.24018 / \mathrm{ejbmr} .2021 .6 .6 .1138$ \\ Sina E. Charandabi \\ LeBow School of Business, Drexel \\ University, Philadelphia, PA, USA. \\ (e-mail: se456@drexel.edu) \\ Kamyar Kamyar * \\ Department of Economics, The Ohio State \\ University, Columbus, OH, USA. \\ (e-mail: Kamyar.5@osu.edu) \\ *Corresponding Author
}

\section{INTRODUCTION}

While the primarily well-known cryptocurrency, Bitcoin, was created by Nakomoto (Nakamoto, 2008), the idea of such secured peer-to-peer blockchain-based digital currency had been around for the last few decades. Cryptographer David Chaum initially introduced the idea in a 1983 paper whose initiative was to enhance the security of credit card transactions through a virtual system (Chaum, 1983) He also founded the first cryptocurrency, DigiCash, which went bankrupt after a decade.

Despite the absence of rapid growth in the first few years after its launch, Bitcoin has been growing extremely rapidly since 2017. It has frequently been experiencing price hikes, therefore attracting numerous investors. Its popularity has grown to a level that in addition to many private companies, a country has recently announced to be accepting it as a form of payment. Not to mention, researchers from central banks have been analyzing and debating on it since at least 2014 .

The major factor distinguishing Bitcoin from other types of currency is its decentralization. That is, unlike other currencies, Bitcoin transactions are not processed and/or supervised by any government. Its money supply increases over time, though not linearly, by a reward process called "mining". Computers engage in solving mathematical equations through brute force, receiving bitcoins as a reward. Indeed, the classic supply and demand laws determine the exchange rate of Bitcoin prices to other currencies.

Similar to any other financial process, bitcoin prices could be predicted by artificial neural network methods. While the history of implementation of artificial neural network methods to predict other financial processes (e.g., stocks prices) is lengthy, the literature of prediction of cryptocurrency prices is not as much due to its novel nature.
Despite, over the last few years a growing number of researchers have attempted to create hybrid models of artificial neural networks that predict the price and/or price volatility of cryptocurrency prices, with a major focus on Bitcoin. This survey paper presents a number of research papers attempting to do so as well as their results. Comparison of methods and results follow in the results section.

\section{THE ISSUE OF VOLATILITY}

As elaborated on throughout the paper, numerous hybrid combinations and configurations of artificial neural network algorithms have been actively used for forecasting time-series data of various natures over the past few decades. Such algorithms often perform with substantial success and efficiency depending on the nature and context of the data. Forecasting techniques have a wide usage among the vast engineering literature, as well as financial engineering and economics. However, a repetitive argument in criticism of using neural networks for financial time-series predictions deals with the volatility of the data (Ghashami et al., 2021).

Accordingly, the volatile nature of the data as well as the dependency of such financial measures on real-life events and shocks, which are hard to predict by nature, play a significant role in determination of stocks market prices. Such criticism is similarly applicable to cryptocurrency exchange rates as well. Ghorbani and Korzeniowski (2020) exemplify such case. We refer to Andersen et al. (2003) for addressing the issue of forecasting realized volatility. They create a framework for forecasting high-frequency time-series data; and due to continuous-time arbitrage-free price processes and the quadratic variation theorem, formal links are established between realized volatility and the conditional covariance 
matrix. The links are further examined using data from spot exchange rates of multiple currencies and are found to perform well through a simple long-memory Gaussian vector autoregressive for logarithmic daily model. While the results are specific to a simple Gaussian autoregressive model and tested for limited data, the results may hold for forecasting any intraday time-series data with high volatility. Though the concept lies beyond the scope of this paper, some similar literature establishes models to predict the volatility of cryptocurrency price data using artificial neural networks.

\section{BASIC MODELS}

Earlier scholar works in price prediction (including but not limited to cryptocurrency) use linear regression optimization methods. Saad et al. (2018) implemented multiple regressionbased models to predict Bitcoin prices using pre-2017 data points. The authors also use data from prices of other cryptocurrencies (Ripple, Litecoin, Dash, Ethereum) and provide a correlation matrix of their prices using the Pearson method.

The methods provided in Saad et al. (2018) are linear regression, random forest, and gradient descent. Data from 2016-2017 is implemented in the models and yields the following results (Table 1). The results include Root Mean Squared Error (RMSE), Mean Absolute Error value (MAE), and accuracy. Any unsatisfactory results may be due to the nature of the aforementioned methods.

\begin{tabular}{cccc} 
TABLE I: RESULTS OF EXPERIMENTS OF THE THREE MODELS USED IN \\
& SAAD ET AL. (2018) & \\
\hline Method & Accuracy & RMSE & MAE \\
\hline Linear Regression & 0.9944 & 0.0113 & 0.0060 \\
Random Forest & 0.9272 & 0.0407 & 0.0115 \\
Gradient Decent & 0.9401 & 0.0369 & 0.0113 \\
\hline
\end{tabular}

\section{IMPLEMENTATION OF LONG-SHORT-TERM-MEMORY (LSTM)}

The majority of papers have analyzed the prediction of Bitcoin prices in an ANN-LSTM setting using different data. Zhengyang et al. (2019) uses Bitcoin price data from August 2015 to June 2019 to do so. The artificial neural networks are trained by gradient based back-propagation algorithm. Relations below depict the mathematical framework for nodes.

where

$$
\text { node }=f\left(\sum_{i=1}^{n} w_{i} x_{i}\right)
$$

$$
f_{\text {node }}=\frac{1}{1+e^{\text {node }}}
$$

Architecture of the LSTM mechanism is given in Fig. 1.

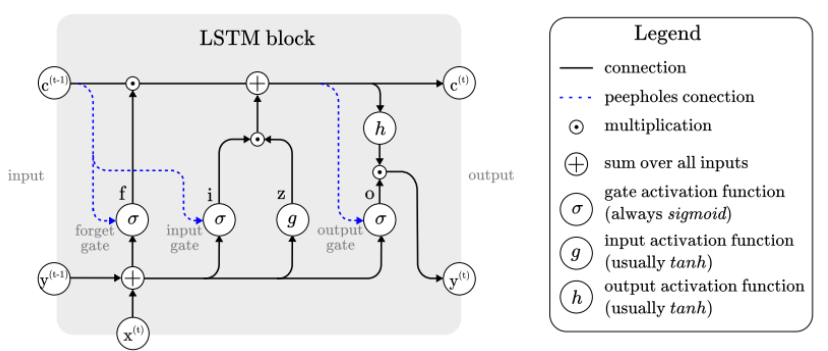

Fig. 1. Architecture of an LSTM block (Van Houdt et al., 2020).
Zhengyang et al. (2019) uses multiple experiments that predict Bitcoin prices separately using ANN-LSTM, as well as a joint experiment of multiple cryptocurrencies. Accordingly, in the single prediction, with 20 nodes per layer and three layers in ANN, and training epoch number of 500 and batch size of 100 in LSTM, the model predicts a RMSE of 209 and a MAE of 99. In the LSTM single prediction, however, the RMSE and MAE values are 3894 and 2789, respectively. In the joint experiment, the resulting RMSE and MAE values are 137 and 30. The authors also argue that model error increases as the predictive time scale is larger.

A similar framework is used in Livieris et al. (2021), where the authors use a Convolutional Neural Networks (CNN) hybrid with LSTM to predict the prices of the three cryptocurrencies with highest market capitalization: Bitcoin (BTC), Ethereum (ETH), and Ripple (XRP). Their experiment implements three years (January 2017 to October 2020) of price data into a multiple-input deep neural network framework. The lags used in the experiment are 7 and 14 days, and the multiple models alter in the number of their convolutional layers (16 and 32, respectively) as well as the number of neurons in their dense layer (64 and 128, respectively). Finally, the MICDL model consists of 3 convolutional layers with 16 filters, each taking an input of a unique time-series data (average pooling of size 2), and a LSTM layer of 50 units. The experiment results are depicted in tables 2 through 4 . Based on the data and further tests, the authors argue that MICDL is the most appropriate model in terms of performance prediction.

TABLE II: PERFORMANCE OF THE EVAluATED MOdELS FOR All BTC

\begin{tabular}{cccccc}
\multicolumn{5}{c}{ DATA (LIVIERIS ET AL. 2021) } \\
\hline Model & Lag & MAE & RMSE & R-squared & Accuracy \\
\hline 1 & 7 & 169.81 & 256.68 & 0.953 & $55.03 \%$ \\
2 & & 169.60 & 256.31 & 0.953 & $53.64 \%$ \\
MICDL & & 170.76 & 257.72 & 0.952 & $53.04 \%$ \\
1 & \multirow{2}{*}{14} & 171.29 & 262.33 & 0.950 & $53.53 \%$ \\
2 & & 170.10 & 256.84 & 0.952 & $52.60 \%$ \\
MICDL & & 171.14 & 257.84 & 0.952 & $51.88 \%$ \\
\hline
\end{tabular}

TABLE III: PERFORMANCE OF THE EVALUATED MODELS FOR ALL ETH DATA (LIVIERIS ET AL. 2021)

\begin{tabular}{cccccc}
\hline Model & Lag & MAE & RMSE & R-squared & Accuracy \\
\hline 1 & & 9.172 & 13.517 & 0.964 & $51.51 \%$ \\
2 & 7 & 9.302 & 13.591 & 0.964 & $48.85 \%$ \\
MICDL & & 9.233 & 13.551 & 0.964 & $50.86 \%$ \\
1 & & 9.309 & 13.657 & 0.964 & $49.57 \%$ \\
2 & \multirow{2}{*}{14} & 9.196 & 13.539 & 0.964 & $50.38 \%$ \\
MICDL & & 9.146 & 13.492 & 0.964 & $51.11 \%$ \\
\hline
\end{tabular}

TABLE IV: PERFORMANCE OF THE EVALUATED MODELS FOR ALL XRP

\begin{tabular}{cccccc}
\multicolumn{5}{c}{ DATA (LIVIERIS ET AL. 2021) } \\
\hline Model & Lag & MAE & RMSE & R-squared & Accuracy \\
\hline 1 & & 0.005 & 0.007 & 0.960 & $48.97 \%$ \\
2 & 7 & 0.005 & 0.007 & 0.958 & $49.61 \%$ \\
MICDL & & 0.005 & 0.007 & 0.958 & $49.07 \%$ \\
1 & & 0.005 & 0.007 & 0.962 & $49.34 \%$ \\
2 & \multirow{2}{*}{14} & 0.006 & 0.009 & 0.936 & $49.23 \%$ \\
MICDL & & 0.007 & 0.009 & 0.953 & $49.23 \%$ \\
\hline
\end{tabular}

Another research paper with prediction models featuring LSTM is Jaquart et al. (2021). The paper focuses on shortterm movement predictions of the cryptocurrency market and attempts the question using an LSTM and GRU (Gated Recurrent Units) approach. Their prediction horizon being 160 minutes, they provide cryptocurrency price data from 2019 
in an extremely volatile period.

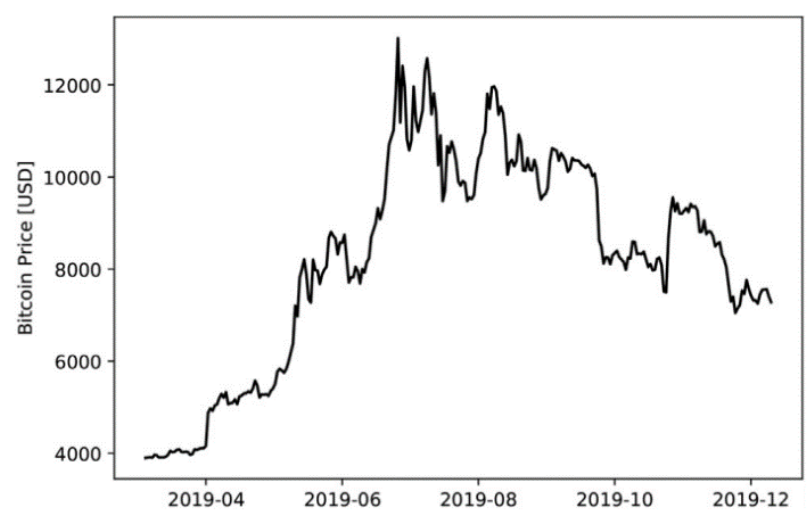

Fig. 2. Bitcoin price volatility in 2019 (Jaquart et al. 2021).

The model implements feedforward, LSTM and GRU, tree-based, and random forest artificial neural network techniques, with LSTM/GRU number of memory blocks of $64,128,256$, and 512. Feedforward neural networks (FNN) process information in a single direct

ion, with three layers of input, a variable number of hidden layers, and an output layer. The activation function in equation 3 determines the result. Here, $g(n)$ is the activation function, $\mathrm{W}(\mathrm{n})$ is the weight matrix for the connections between layer $n-1$ and layer $n$, and $b(n)$ is the bias for layer $n$. The recurrent neural network model used is depicted in Fig. 3 . Finally, the accuracy results are given in Table V.

$$
a(n)=g(n)\left(W(n)^{T} a(n-1)+b(n)\right)
$$
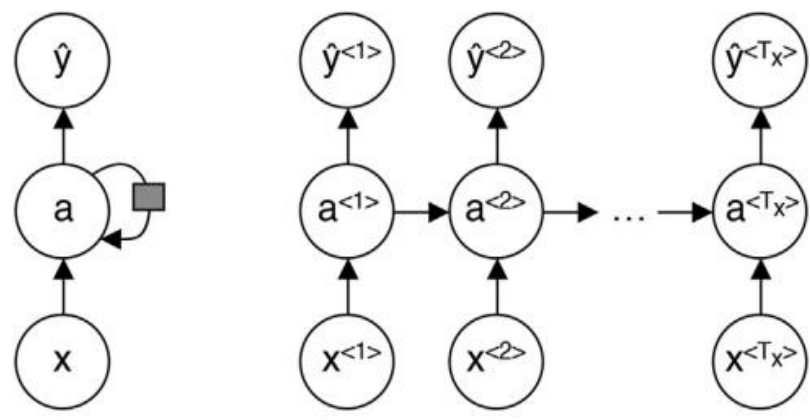

Fig. 3. RNN Structure. Jaquart et al. (2021).

TABLE V: ACCURACY OVERVIEW AMONG METHODS JAQUART ET AL.

\begin{tabular}{ccccc}
\multicolumn{5}{c}{$(2021)}$. \\
Model & $\begin{array}{c}\text { 1-Min } \\
\text { Pred. }\end{array}$ & $\begin{array}{c}\text { 5-Min } \\
\text { Pred. }\end{array}$ & $\begin{array}{c}\text { 15-Min } \\
\text { Pred. }\end{array}$ & $\begin{array}{c}\text { 60-Min } \\
\text { Pred. }\end{array}$ \\
\hline GRU & 0.518411 & 0.524562 & 0.536490 & 0.556653 \\
LSTM & 0.519286 & 0.524931 & 0.531967 & 0.560067 \\
FNN & 0.509438 & 0.521988 & 0.520820 & 0.529587 \\
LR & 0.511272 & 0.517926 & 0.519595 & 0.538552 \\
GBC & 0.511093 & 0.529268 & 0.537282 & 0.557026 \\
RF & 0.511947 & 0.526662 & 0.534641 & 0.556356 \\
E (All) & 0.514626 & 0.526092 & 0.537863 & 0.557579 \\
\hline
\end{tabular}

Using the accuracies and binomial distribution, the authors provide the probability of prediction models to have a true accuracy of $50 \%$ in Table V. In conclusion, they argue that RNN and GBC models are the most appropriate for predicting the short-term bitcoin market.
TABLE VI: BINOMIAL PROBABILITIES FOR TRUE MODEL ACCURACY OF

\begin{tabular}{ccccc}
\multicolumn{5}{c}{$50 \%(\mathrm{JAQUART}$ ET AL. } \\
\hline \multirow{2}{*}{ Model } & $\begin{array}{c}\text { 1-Min } \\
\text { Pred. }\end{array}$ & $\begin{array}{c}\text { 5-Min } \\
\text { Pred. }\end{array}$ & $\begin{array}{c}15-\mathrm{Min} \\
\text { Pred. }\end{array}$ & $\begin{array}{c}\text { 60-Min } \\
\text { Pred. }\end{array}$ \\
\hline GRU & $4.18 \mathrm{E}-30$ & $5.66 \mathrm{E}-52$ & $6.19 \mathrm{E}-11$ & $8.44 \mathrm{E}-265$ \\
LSTM & $7.65 \mathrm{E}-33$ & $1.75 \mathrm{E}-53$ & $1.99 \mathrm{E}-86$ & $2.28 \mathrm{E}-297$ \\
FNN & $3.08 \mathrm{E}-09$ & $4.64 \mathrm{E}-42$ & $7.17 \mathrm{E}-38$ & $7.02 \mathrm{E}-74$ \\
LR & $1.92 \mathrm{E}-12$ & $1.26 \mathrm{E}-28$ & $8.99 \mathrm{E}-34$ & $6.90 \mathrm{E}-124$ \\
GBC & $4.18 \mathrm{E}-12$ & $6.67 \mathrm{E}-73$ & $9.47 \mathrm{E}-12$ & $2.93 \mathrm{E}-268$ \\
RF & $9.31 \mathrm{E}-14$ & $7.30 \mathrm{E}-61$ & $4.23 \mathrm{E}-10$ & $4.75 \mathrm{E}-262$ \\
E (All) & $1.05 \mathrm{E}-19$ & $2.22 \mathrm{E}-58$ & $2.40 \mathrm{E}-12$ & $1.94 \mathrm{E}-273$ \\
\hline
\end{tabular}

\section{IMPLEMENTATION OF GENERAL ALGORITHMS}

A subgroup of literature attempt to predict cryptocurrency prices using hybrid techniques of General Algorithms. Here, Radityo et al. (2017), and Sin et al. (2017) are briefly discussed. The former employs backpropagation neural network (BPNN), genetic algorithm neural network (GANN), genetic algorithm, backpropagation neural network (GABPNN), and neuro-evolution of augmenting topologies (NEAT). The complete results of experiments in Radityo et al. (2017) are given in Table VII.

TABLE VII: RESULTS OF BPNN, GANN, GABPNN, NEAT EXPERIMENTS

\begin{tabular}{ccc}
\multicolumn{3}{c}{ (RADITYO ET AL. 2017). } \\
\hline Method & MAPE & Training Time $(\mathrm{s})$ \\
\hline BPNN & $1.998 \pm 0.038$ & $347 \pm 63$ \\
GANN & $4.461 \pm 0.49$ & $467 \pm 345$ \\
GABPNN & $1.883 \pm 0.066$ & $1539 \pm 558$ \\
NEAT & $2.175 \pm 0.096$ & $470 \pm 363$ \\
\hline
\end{tabular}

In Sin et al. (2017), a hybrid model of Genetic Algorithm based Selective Neural Network Ensemble (GASEN) is implemented to predict the next day change in the direction of the price of Bitcoin. Used data spans 2 years of time-series Bitcoin prices, and the number of epochs for each MLP was set as 30. The authors report the classification function to be represented by relation 4. Accordingly, the results are satisfactory in the sense that using the certain given datapoints, a simulation of investment would yield positive profit. They further report that GASEN was able to perform with a "consistent accuracy of around 58\% to 63\%" (Sin et al. 2017).

$$
y=\frac{2}{1+e^{-2 x}}-1
$$

\section{GREY SYSTEM THEORY}

Grey system theory is an optimization method using a firstorder differential equation to model the time-series data trend of nonlinear nature. GM (n, m) refers to a grey prediction model with $n$ degrees of differential equations and $m$ variables. Faghih Mohammadi Jalali et al. (2020) uses a GM $(1,1)$, namely a classical grey model to predict Bitcoin prices and measure efficiency of their proposed method. In order to more accurately depict results, they divide the data into 8 parts and attempt to compute predictions separately. The first five parts each consist of 4 days of time-series data, and the latter three parts each consist of 6 months of time-series data. For the former set of groups, the resulting MAPE values are $0.34 \%, 1.66 \%, 1.04 \%, 1.13 \%$, and $0.22 \%$, respectively. For the latter set of groups, the resulting MAPE values are 
$13.67 \%, 15.60 \%$, and $9.45 \%$ respectively. Due to the strong MAPE resulting values, the authors find the results significant. They further argue that non-statistical methods such as grey system theory are suitable for prediction of such prices.

\section{DISCUSSION AND CONCLUSION}

As shown in the previous sections, the purpose of this survey paper is to present and compare multiple research papers that employed multiple artificial neural network-based approaches to predict cryptocurrency prices. Noting the pros and cons of each of the methods presented (in terms of time elapsed, prediction accuracy, MAPE, and R-squared), it appears that in a holistic manner the methods used in Jaquart et al. (2021), and the MICDL method in Livieris et al. (2021) are of significant efficiency.

Due to the novel nature of this literature, all papers written in the area span solely to the last few years. Therefore, it's expected that many additional papers be written in the near future. There exist many aspects of cryptocurrency price forecasting with no existent research papers at the time. One area of possible further work, for instance, is to focus solely on implementing data from less volatile cryptocurrencies (e.g., Ethereum, XRP, Chainlink, etc.) into the same frameworks. Indeed, many other frameworks could be implemented to forecast and/or measure the prediction efficiencies as well.

It should also be noted that another subgroup of related literature uses some similar and/or the same methods presented throughout this survey to predict the volatility, rather than future prices, of cryptocurrencies. This lies beyond the scope of this paper and is a possible area of research and/or another survey paper of existing literature.

Due to the prominent recent increase in the popularity and price of cryptocurrencies (including but not limited to Bitcoin), introduction to the history and technicalities of cryptocurrency is of essential importance to policymakers and other economic agents. The first transaction using bitcoins was to buy a pizza in 2011, which would have been worth over $\$ 5$ million today. With such quick turnover, agents need to catch up quickly (Charandabi et al. 2021).

\section{REFERENCES}

Andersen, T.G., Bollerslev, T., Diebold, F., Labys, P. (2003) "Modeling and forecasting realized volatility". Econometrica 71, 579-625.

Bonini, S. and Taatian, A. (2021) "Dual Holding and Bank Risk" http://dx.doi.org/10.2139/ssrn.3815293.

Charandabi, S. E., \& Kamyar, K. (2021) "Using A Feed Forward Neural Network Algorithm to Predict Prices of Multiple Cryptocurrencies". European Journal of Business and Management Research, 6(5), 15-19. https://doi.org/10.24018/ejbmr.2021.6.5.1056.

Charandabi, S. E., Ghashami, F., Kamyar, K. (2021) "US-China Tariff War: A Gravity Approach,” Business and Economic Research, Macrothink Institute. 11 (3). https://doi.org/10.5296/ber.v11i3.18757.

Chaum, D. (1983) "Blind signatures for untraceable payments". In Chaum, D., Rivest, R. L., and Sherman, A.T. editors, Advances in Cryptology - Proceedings of CRYPTO '82, pp. 199-203. Plenum Press.

Faghih Mohammadi Jalali, M., Heidari, H. (2020) "Predicting changes in Bitcoin price using grey system theory". Financ Innov 6, 13. https://doi.org/10.1186/s40854-020-0174-9.

Ghashami, F., \& Kamyar, K. (2021) "Performance Evaluation of ANFIS and GA-ANFIS for Predicting Stock Market Indices". International
Journal of Economics and Finance. https://doi.org/10.5539/ijef.v13n7p1.

Ghashami, F., Kamyar, K., \& Riazi, S. A. (2021) "Prediction of Stock Market Index Using a Hybrid Technique of Artificial Neural Networks and Particle Swarm Optimization". Applied Economics and Finance, 8, 1. https://doi.org/10.11114/aef.v8i3.5195.

Ghorbani, N. and Korzeniowski, A. "Adaptive Risk Hedging for Call Options under Cox-IngersollRoss Interest Rates". Journal of Mathematical Finance, 10, 697-704, 2020. https://doi.org/10.4236/jmf.2020.104040.

Jaquart, P., Dann, D., \& Weinhardt, C. (2021) "Short-term bitcoin market prediction via machine learning." The Journal of Finance and Data Science, 7, pp. 45-66. https://doi.org/10.1016/j.jfds.2021.03.001.

Jay, P., Kalariya, V., Parmar, P., Tanwar, S., Kumar, N., and Alazab, M. (2020) "Stochastic Neural Networks for Cryptocurrency Price Prediction," in IEEE Access, 8, 82804-82818. doi: 10.1109/ACCESS.2020.2990659.

Kamyar, K. (2019) "Effect of Unemployment Length on Employment Expectations". Undergraduate Economic Review.

Korzeniowski, A. and Ghorbani, N. (2021) "Put Options with Linear Investment for Hull-White Interest Rates". Journal of Mathematical Finance, 11, 152-162. https://doi.org/10.4236/jmf.2021.111007.

Kousha, P., Sankarapandian Dayala Ganesh Ram, K. R., Kedia, M., Subramoni, H., Jain, A., Shafi, A., Panda, D., Dockendorf, T., Na, H., \& Tomko, K. Inam (2021) "Cross-stack profiling and analysis of Communication in MPI-based applications". Practice and Experience in Advanced Research Computing. https://doi.org/10.1145/3437359.3465582.

Liu, S., Tao, L., Xie, N., and Yang, Y. (2015) "On the new model system and framework of grey system theory," 2015 IEEE International Conference on Grey Systems and Intelligent Services (GSIS), 1-11. doi: 10.1109/GSIS.2015.7301810.

Livieris, I.E., Kiriakidou, N.; Stavroyiannis, S.; Pintelas, P. (2021) “An Advanced CNN-LSTM Model for Cryptocurrency Forecasting". Electronics 10, 287.

DOI: https://doi.org/10.3390/electronics10030287.

Nakamoto, S. (2008) "Bitcoin: A Peer-to-Peer Electronic Cash System." https://bitcoin.org/bitcoin.pdf.

Radityo, A., Munajat, Q., and Budi, I. (2017) "Prediction of Bitcoin exchange rate to American dollar using artificial neural network methods," 2017 International Conference on Advanced Computer Science and Information Systems (ICACSIS), 433-438. DOI: 10.1109/ICACSIS.2017.8355070.

Saad, M., and Mohaisen, A. (2018) "Towards characterizing blockchainbased cryptocurrencies for highly-accurate predictions," in Proc. IEEE Conf. Comput. Commun. Workshops (INFOCOM WKSHPS), Apr. 2018, 1-7.

Shi, N. (2016) “A new proof-of-work mechanism for bitcoin”. Financ Innov 2, 31. https://doi.org/10.1186/s40854-016-0045-6.

Sin, E., and Wang, L. (2017) "Bitcoin price prediction using ensembles of neural networks," 2017 13th International Conference on Natural Computation, Fuzzy Systems and Knowledge Discovery (ICNCFSKD), pp. 666-671. doi: 10.1109/FSKD.2017.8393351.

Van Houdt, G., Mosquera, C. \& Nápoles, G. (2020) "A review on the long short-term memory model". Artif Intell Rev 53, 5929-5955. https://doi.org/10.1007/s10462-020-09838-1.

Yermack, D. (2015) "Is Bitcoin a real currency? An economic appraisal”, Handbook of digital currency, pp. 31-43.

Zhengyang, W., Xingzhou, L., Jinjin, R., and Jiaqing, K. (2019) "Prediction of Cryptocurrency Price Dynamics with Multiple Machine Learning Techniques". In Proceedings of the 2019 4th International Conference on Machine Learning Technologies (ICMLT 2019). Association for Computing Machinery, New York, NY, USA, pp 15-19. DOI: https://doi.org/10.1145/3340997.3341008. 